\title{
T-Helper 17 Cell/Regulatory T-Cell Imbalance in COPD Combined with T2DM Patients
}

\author{
Cheng Wang' \\ Hong Wang ${ }^{2}$ \\ Luming Dai' \\ Jianqing Zhang' \\ Lizhou Fang' \\ Ling Liu' \\ Weiping Fu' \\ Dang Tang ${ }^{3}$
}

'Second Department of Respiratory Medicine, The First Affiliated Hospital of Kunming Medical University, Kunming, 650032, People's Republic of China; ${ }^{2}$ Department of Burns Surgery, The Second Affiliated Hospital of Kunming Medical University, Kunming, 650I0I, People's Republic of China; ${ }^{3}$ First Department of Neurosurgery, The First Affiliated Hospital of Kunming Medical University, Kunming, 650032, People's Republic of China
Background: Chronic obstructive pulmonary disease (COPD) is often combined with type 2 diabetes mellitus (T2DM) in clinical, and with poor prognosis. In recent years, research shows that inflammation is a common characteristic of COPD and T2DM. T-helper 17 cell (Th17)/regulatory T-cell (Treg) balance controls inflammation and may be important in the pathogenesis of COPD combined with T2DM patients. This study investigated the characteristics of Th17, Treg and related inflammatory factors in COPD combined with T2DM patients and the potential mechanism.

Methods: Application of flow cytometry technology, real-time fluorescent quantitative PCR and ELISA to detect the changes in peripheral blood of Th17 and Treg number and the expression of key transcription factors and related cytokines in COPD combined T2DM patients were performed.

Results: Patients with COPD combined with T2DM revealed significant increase in peripheral Th17, Th17 related cytokines (IL-17A, IL-17F, IL-21, IL-23, IL-6) and transcription factor (ROR $\gamma \mathrm{t}$ ) levels and significant decrease in Treg, Treg-related cytokines (IL-10, TGF $\beta 1$ ) and transcription factor (Foxp3) as compared with patients with COPD, T2DM and healthy controls.

Conclusion: Th17/Treg functional imbalance exists in patients with COPD combined with T2DM, indicating a potential role of Th17/Treg imbalance in the formation and progression of COPD combined with T2DM.

Keywords: COPD combined with T2DM, Th17/Treg, cytokine, transcription factor

\section{Introduction}

Chronic obstructive pulmonary disease (COPD) is a common disease of the respiratory system. With increased air pollution, the prevalence of COPD is rising, and it is expected to more than 4.5 million people died of COPD every year since 2030. ${ }^{1}$ COPD not only affects the respiratory system, but also involves the whole body, among which the impact on the endocrine system is causing more and more researchers' attention. COPD combined with type 2 diabetes mellitus (T2DM) is a high incidence of clinical complications, according to statistics, about $10 \%$ in patients with diabetes merged COPD. ${ }^{2}$ Studies have shown that normal people the prevalence of diabetes is $10.5 \%$, and the prevalence of COPD patients with diabetes is as high as $18.7 \% .^{3}$ COPD combined with T2DM have higher hospital readmission rate, for T2DM will increase progression, poor prognosis and mortality in CDPD patients. ${ }^{4,5}$

T2DM can affect the structure of lung tissue and lung function, make the connective tissue glycosylation, reduce pulmonary elastic retraction force, promote
Correspondence: Dang Tang

Email tangdangdemao@।26.com 
inflammation, cause pulmonary capillary damage and pulmonary ventilation/blood flow ratio imbalance, further lead to hypoxia and vicious cycle. ${ }^{6}$ Moreover, hyperglycemia can stimulate bacterial growth or promote interaction between respiratory epithelial cells and bacteria, which makes patients susceptible to infection, and promote the pathological progress of COPD. ${ }^{7}$ At present, more and more scholars believe that the cause of T2DM and COPD is associated with cytokines mediated inflammatory response. The rise of inflammatory mediators in the T2DM patients shows the consistency and comparability with COPD patients. ${ }^{8}$ These studies demonstrate that although COPD and T2DM are different diseases, there is some correlation between them in terms of pathological mechanism. The current study shows that critical roles for Th17 and Treg subsets are involved in immunopathologic inflammation. ${ }^{9}$ The imbalance between producing Th17 cells that act as pro-inflammatory effectors and Treg cells that act as effector cells in peripheral tolerance and downregulation of inflammation may contribute to the progression of inflammation. ${ }^{10}$ Th17/Treg homeostasis is a novel immunotherapy approach for chronic inflammatory disease. However, the role of Th17-Treg axis and immune balance in COPD combined with T2DM patients remains unexplored.

In the current study, we examined the frequency of peripheral blood Th17 and Tregs, the relative levels of retinoic acid orphan receptor $\gamma \mathrm{t}(\mathrm{ROR}-\gamma \mathrm{t})$ and Forkhead box p3 (Foxp3) mRNA transcripts, and measured the levels of associated serum inflammatory cytokines in COPD combined with T2DM patients. Also, we analyzed the potential association of the values of the balance of Th17/Treg cells, transcription factors, cytokines and clinical indexes in these patients.

\section{Materials and Methods}

\section{Study Design and Subjects}

30 healthy control subjects, 36 patients with acute exacerbation of COPD (C group), 35 patients with T2DM (D group) and 32 patients with COPD combined with T2DM $(\mathrm{C}+\mathrm{D}$ group) were recruited from The First Affiliated Hospital of Kunming Medical University. Patients with acute exacerbation of COPD symptoms were diagnosed with COPD according to the Global Initiative for Chronic Obstructive Lung Disease (GOLD). T2DM was diagnosed according to WHO1999 diabetes classification and diagnostic criteria. Written informed consent was obtained from each subject and the protocol was approved by the Ethics Committee of The First Affiliated Hospital of Kunming Medical University and adhered to the tenets of the Declaration of Helsinki. Patients were excluded if they had suffered from serious cardiovascular disease, malignant tumors, cognitive impairment, immunodeficiency or other chronic inflammatory diseases that may have influenced the results of the study.

\section{Sample Collection and Processing}

We collected 5-10 mL of peripheral blood samples from each subject, and isolated peripheral blood mononuclear cells (PBMCs) using lymphocyte separation medium. PBMCs were prepared by Ficoll density gradient centrifugation for analysis of flow cytometry (FCM) and quantitative real-time PCR (qRT-PCR), and serum was obtained after centrifugation for enzyme-linked immunosorbent assay (ELISA) measurements.

\section{Flow Cytometry Analysis of ThI7 and}

\section{Tregs}

PBMCs were isolated from individual participants and stained with fluorescein isothiocyanate (FITC)-labeled antihuman CD4, phycoerythrin cyanidin 5 (PE-Cy5)-labeled anti-human CD25 for $30 \mathrm{~min}$, fixed and permeabilized with the fixation and permeabilization buffer, followed by staining with PE-labeled anti-human Foxp3 or isotype control (BD Pharmingen, San Diego, CA, USA), to determine the frequency of $\mathrm{CD} 4^{+} \mathrm{CD} 25^{+}$Foxp $3^{+}$Tregs. Some PBMCs were stimulated with phorbol 12-myristate 13-acetate and ionomycin in the presence of brefeldin $\mathrm{A}$ in $10 \%$ fetal calf serum at $37^{\circ} \mathrm{C}$ for $4 \mathrm{~h}$. Then, the cells were harvested and stained with FITC-labeled anti-human CD3 and APClabeled anti-human $\mathrm{CD} 8$ at $4^{\circ} \mathrm{C}$ for $30 \mathrm{~min}$, followed by fixing, permeabilization, and staining with PE-labeled antihuman IL-17A (BD Pharmingen, San Diego, CA, USA), to determine the frequency of $\mathrm{CD}^{+} \mathrm{CD} 8^{-} \mathrm{IL}-17^{+}$Th17 cells. Flow cytometry was performed on a fluorescence-activated cell sorter (FACS) Canto II (BD Biosciences) and analyzed with CellQuest software (BD Corporation).

\section{qRT-PCR Analysis}

Total RNA was extracted from PBMC samples with Trizol reagent (Invitrogen, Carlsbad, CA, USA). Quantity and quality was determined by Nanodrop spectrophotometer with OD $260 \mathrm{~nm}$ for measuring concentration and 260/280 ratio for assessing the purity. The cDNA was synthesized with the 
reverse transcription kit (GeneCopoeia, Guangzhou, China). All reactions were performed using SYBR Green.

Master mix reagents on the ABI Prism 7900 Sequence Detection System (Applied Biosystems, Carlsbad, CA, USA) and the specific primers. The forward primer of GAPDH was 5'-CGCTGAGTACGTCGTGGAGTC-3' and the reverse primer was 3'-GCTGATGATCTTGAGGCTGTTGTC-5'. The forward primer of Foxp3 was 5'-AAGGAAAGG AGGATGGACGAA-3' and the reverse primer was $3^{\prime}-\mathrm{CAG}$ GCAAGACAGTGGAAACCT-5'. The forward primer of ROR- $\gamma \mathrm{t}$ was 5'-AGTCGGAAGGCAAGATCAGA-3' and the reverse primer was 3'-CAAGAGAGGTTCTGGGCAAG-5'. Thermal cycling of PCR system ( $20 \mu \mathrm{L}$ in total) comprised 10 $\mu \mathrm{L}$ of SYBR Green, $0.4 \mu \mathrm{L}$ of forward primer, $0.4 \mu \mathrm{L}$ of reverse primer, $2 \mu \mathrm{L}$ of cDNA, and corresponding $\mathrm{ddH}_{2}$ O. mRNA expression level was normalized to the level of GAPDH housekeeping genes. Relative gene expression was calculated using the $2^{-\Delta \Delta \mathrm{Ct}}$ method. Data were expressed as the fold change of the controls.

\section{ELISA Analysis for Inflammatory Biomarkers}

The concentrations of serum inflammatory cytokines were determined with sandwich ELISA kits according to the manufacturer's protocols for IL-17A (PK-EL -62,719), IL-17F (PK-EL-62,718) (Promo Cell,
Heidelberg, Germany), IL-21 (bsk00366), IL-23 (bsk00368), IL-6 (bsk00040), IL-10 (bsk00044) (Bioss, Beijing, China), TGF- $\beta 1$ (MM-0367H1) (DiNing, Beijing, China). In the end of this operation, pore plates were detected absorbance at the wavelength of $450 \mathrm{~nm}$ with microplate reader (Thermo Fisher Scientific, Waltham, MA, USA), and drew a standard curve, calculated the OD value of the samples.

\section{Statistical Analysis}

SPSS 20.0 software was used for statistical analysis. Student's $t$-test was used to compare two group experiments, and one-way analysis of variance (ANOVA) followed by Bonferroni post hoc tests for multiple comparisons. $\chi^{2}$ test was used to compare categorical variables. Data are expressed as the means \pm standard deviation (SD). $\mathrm{P}<0.05$ was considered statistically significant.

\section{Results}

\section{Baseline Characteristics of Study Participants}

The characteristics of the participants enrolled in the study are summarized in Table 1. There was no significant difference among healthy control subjects ( $\mathrm{H}$ group), COPD (C group), T2DM (D group) and COPD

Table I Demographics and Clinical Characteristics in the Each Group of Subjects

\begin{tabular}{|c|c|c|c|c|c|}
\hline Variables & $\begin{array}{c}\text { Healthy Control } \\
\text { Group }\end{array}$ & $\begin{array}{l}\text { COPD } \\
\text { Group }\end{array}$ & $\begin{array}{l}\text { T2DM } \\
\text { Group }\end{array}$ & $\begin{array}{l}\text { COPD+T2DM } \\
\text { Group }\end{array}$ & P-value \\
\hline Subjects (No.) & 30 & 36 & 35 & 32 & \\
\hline Age (year) & $65.82 \pm 9.37$ & $70.24 \pm 8.14$ & $68.17 \pm 8.47$ & $66.30 \pm 10.72$ & 0.189 \\
\hline Gender (Male/female) & $21 / 9$ & $28 / 8$ & $24 / 11$ & $25 / 7$ & 0.725 \\
\hline BMI $\left(\mathrm{kg} / \mathrm{m}^{2}\right)$ & $24.34 \pm 3.82$ & $23.83 \pm 4.95$ & $26.43 \pm 4.21$ & $24.86 \pm 3.57$ & 0.062 \\
\hline Smoking rate, $\mathrm{n}(\%)$ & $6(20 \%)$ & 15 (4I.67\%) & $8(22.86 \%)$ & $13(40.63 \%)$ & 0.368 \\
\hline Fasting blood glucose $(\mathrm{mmol} / \mathrm{L})$ & $4.39 \pm 1.12$ & $4.43 \pm 0.99$ & $8.96 \pm 3.57^{* * \#}$ & $7.87 \pm 3.02^{* * \#}$ & $<0.001$ \\
\hline $\begin{array}{l}\text { 2h postprandial blood glucose (mmol/ } \\
\mathrm{L})\end{array}$ & $6.24 \pm 2.17$ & $6.83 \pm 2.46$ & $14.52 \pm 4.98^{* * \#}$ & $|3.6| \pm 4.55^{* * \#}$ & $<0.001$ \\
\hline HbAlc (\%) & $5.64 \pm 1.72$ & $6.08 \pm 1.65$ & $8.34 \pm 2.02^{* * \#}$ & $8.70 \pm 2.36 * \#$ & $<0.001$ \\
\hline FEVI/FVC\% & $89.25 \pm 9.43$ & $51.57 \pm 10.61 * *$ & $85.64 \pm 11.32^{\# \#}$ & $43.24 \pm 8.06^{* * \# \Delta \Delta}$ & $<0.001$ \\
\hline FEVI\% predicted & $83.12 \pm 8.46$ & $50.87 \pm 8.24 * *$ & $79.05 \pm 9.15^{\# \#}$ & $42.37 \pm 9.30 * \# \Delta \Delta$ & $<0.001$ \\
\hline
\end{tabular}

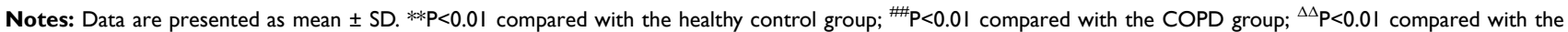
T2DM group.

Abbreviations: BMI, body mass index; FEVI, forced expiratory volume in I second; FVC, forced vital capacity. 
combined with $\mathrm{T} 2 \mathrm{DM}(\mathrm{C}+\mathrm{D}$ group) with respect to age, gender, BMI and smoking $(\mathrm{P}>0.05)$. Regarding fasting blood glucose, $2 \mathrm{~h}$ postprandial blood glucose and $\mathrm{HbA} 1 \mathrm{c}$ were significantly higher in $\mathrm{D}$ group and $\mathrm{C}$ $+\mathrm{D}$ group compared with $\mathrm{H}$ group and $\mathrm{C}$ group $(\mathrm{P}<0.01)$. FEV1\% predicted and FEV1/FVC $\%$ in $\mathrm{H}$ group and $\mathrm{D}$ group were significantly higher compared with $\mathrm{C}$ group and $\mathrm{C}+\mathrm{D}$ group $(\mathrm{P}<0.01)$, and change of FEV1\% predicted and FEV1/FVC \% between $\mathrm{H}$ group and $\mathrm{D}$ group was no significant difference $(\mathrm{P}>0.05)$.

\section{Imbalance of Circulating ThI7 and Tregs in COPD, T2DM and COPD Combined with T2DM Patients}

Flow cytometry analysis indicated that the percentages of Th17 cells in total $\mathrm{CD}^{+} \mathrm{T}$ lymphocytes in the COPD, T2DM and COPD combined with T2DM patients were significantly higher than those in the healthy controls $(\mathrm{P}<0.01)$. The frequency of circulating Th17 cells in the patients with COPD combined with T2DM was significantly higher than those in the patients with COPD and T2DM. It suggests that the increase of Th17 cells in peripheral blood is a common factor for the occurrence and development of COPD and T2DM. Meanwhile, the percentages of Treg cells in the total $\mathrm{CD}^{+}$ $\mathrm{T}$ lymphocytes in the T2DM and COPD combined with T2DM patients were significantly lower than those in the healthy controls and COPD patients $(\mathrm{P}<0.01)$, but there was no significant difference between healthy controls and COPD patients $(\mathrm{P}>0.05)$.
In addition, the ratios of the percentages of Th17 to Tregs in the COPD, T2DM and COPD combined with T2DM patients were significantly higher than those in the healthy controls $(\mathrm{P}<0.01)$. Moreover, the ratios of the percentages of Th17 to Tregs in the COPD combined with T2DM patients were significantly higher than those in the COPD and T2DM patients $(\mathrm{P}<0.01)$ (Table 2, Figures 1-3). In general, the imbalance of Th17 and Tregs in the COPD combined with T2DM patients was considered the most serious.

\section{Change of $\mathrm{CD}^{+} \mathrm{T}$ Cells Specific Transcription Factor Foxp3 and ROR $\gamma \mathrm{t}$}

Expression of Foxp3 and ROR $\gamma \mathrm{t}$ mRNA transcripts in PBMCs from the different groups of subjects were assessed by quantitative RT-PCR. We found that the relative levels of ROR $\gamma \mathrm{t}$ mRNA transcripts in the COPD combined with T2DM patients were significantly higher than that in the healthy controls, COPD and T2DM patients $(\mathrm{P}<0.01)$, levels of ROR $\gamma t$ in the COPD and T2DM patients were also significantly higher than that in the healthy controls $(\mathrm{P}<0.01)$. While the relative levels of Foxp3 mRNA transcripts in the COPD combined with T2DM patients were significantly lower than those in the healthy controls, COPD and T2DM patients $(\mathrm{P}<0.01)$, levels of Foxp3 in the COPD and T2DM patients were also significantly lower than that in the healthy controls $(\mathrm{P}<0.01)$. With respect to ROR $\gamma$ t and Foxp3 levels, there was no significant difference between COPD and T2DM patients $(\mathrm{P}>0.05)$ (Table 2, Figure 4). The results indicate that the imbalance of peripheral blood Th17 and Tregs in COPD with T2DM patients.

Table 2 Measurement Indicators in the Each Group of Subjects

\begin{tabular}{|c|c|c|c|c|c|c|}
\hline & Healthy Control Group & COPD Group & T2DM Group & COPD+T2DM Group & F-value & P-value \\
\hline ThI7 (CD4+T\%) & $0.568 \pm 0.325$ & $1.365 \pm 0.673 * *$ & $1.230 \pm 0.80 I^{* *}$ & $2.127 \pm 0.917^{* * \# \Delta \Delta}$ & 24.553 & $<0.001$ \\
\hline Treg (CD4 $\left.{ }^{+} \mathrm{T} \%\right)$ & $3.955 \pm 0.937$ & $3.704 \pm 1.164$ & $1.835 \pm 0.872^{* * \# \#}$ & $1.822 \pm 0.819^{* * \# \#}$ & 12.085 & $<0.001$ \\
\hline ThI7/Treg & $0.172 \pm 0.093$ & $0.506 \pm 0.247^{* *}$ & $0.659 \pm 0.28 \mathrm{I} * *$ & I. $157 \pm 0.563^{* * \# \Delta \Delta}$ & 45.502 & $<0.001$ \\
\hline $\mathrm{ROR} \gamma \mathrm{t}$ & $1.000 \pm 0.000$ & $10.800 \pm 1.175^{* *}$ & $9.684 \pm 1.505^{* *}$ & $25.670 \pm 5.117^{* * \# \Delta \Delta \Delta}$ & $14.1 \mid$ & $<0.001$ \\
\hline Foxp3 & $1.000 \pm 0.000$ & $0.718 \pm 0.048 * *$ & $0.748 \pm 0.014^{* *}$ & $0.435 \pm 0.059^{* * \# \Delta \Delta}$ & 73.28 & $<0.001$ \\
\hline IL-I7A (pg/mL) & $6.894 \pm 0.538$ & $9.995 \pm 1.679 * *$ & $10.194 \pm 1.868^{* *}$ & $12.519 \pm 1.754^{* * \# \Delta \Delta \Delta}$ & 26.892 & $<0.001$ \\
\hline IL-I7F (pg/mL) & $2.606 \pm 0.907$ & $4.120 \pm 1.296^{* *}$ & $4.529 \pm 1.319 * *$ & $6.356 \pm 1.508^{* * \# \# \Delta \Delta}$ & 45.034 & $<0.001$ \\
\hline IL-2I (pg/mL) & $3.267 \pm 1.267$ & $5.111 \pm 1.093^{* *}$ & $4.339 \pm 2.087^{*}$ & $7.993 \pm 2.198^{* * \# \Delta \Delta \Delta}$ & 43.193 & $<0.001$ \\
\hline $\mathrm{IL}-23(\mathrm{pg} / \mathrm{mL})$ & $8.507 \pm 2.70 \mathrm{I}$ & $16.558 \pm 4.652 * *$ & $14.534 \pm 5.507^{* *}$ & $20.040 \pm 3.956^{* * \# \Delta \Delta \Delta}$ & 37.595 & $<0.001$ \\
\hline IL-6 (pg/mL) & $19.257 \pm 3.562$ & $25.248 \pm 4.60 \mathrm{I} * *$ & $24.307 \pm 4.291 * *$ & $33.5 \mid 4 \pm 5.313^{* * \# \Delta \Delta \Delta}$ & 19.344 & $<0.001$ \\
\hline IL-I0 (pg/mL) & $10.876 \pm 6.809$ & $7.868 \pm 3.916^{*}$ & $8.084 \pm 3.219 * *$ & $4.913 \pm 1.241^{* * \# \Delta \Delta}$ & 10.417 & $<0.001$ \\
\hline TGF- $\beta I(p g / m L)$ & $25.498 \pm 5.021$ & $20.704 \pm 5.807^{* *}$ & $19.008 \pm 5.477^{* *}$ & $15.142 \pm 4.065^{* * \# \Delta \Delta \Delta}$ & 21.414 & $<0.001$ \\
\hline
\end{tabular}

Notes: Data are presented as mean $\pm S D$. $* P<0.05, * * P<0.01$ compared with the healthy control group; ${ }^{\#} P<0.01$ compared with the COPD group; ${ }^{\triangle \Lambda} \mathrm{P}<0.01$ compared with the T2DM group. GAPDH was used as internal reference to detect the statistical results of ROR $\gamma \mathrm{t}$ and Foxp3. 
A

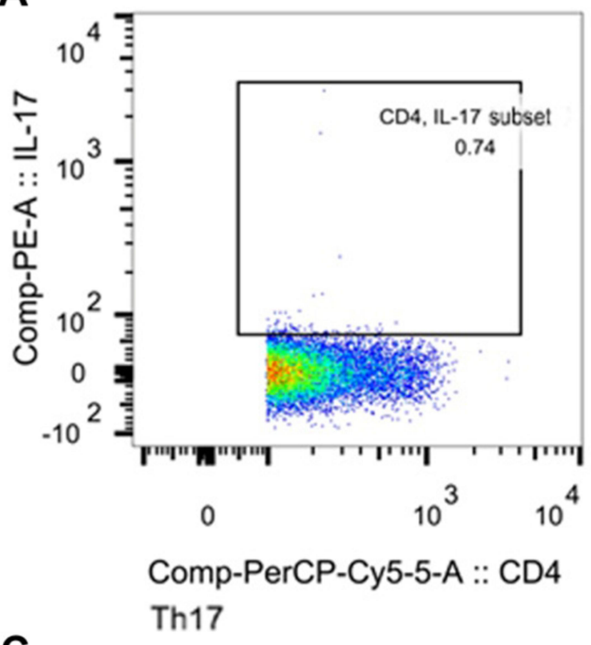

C

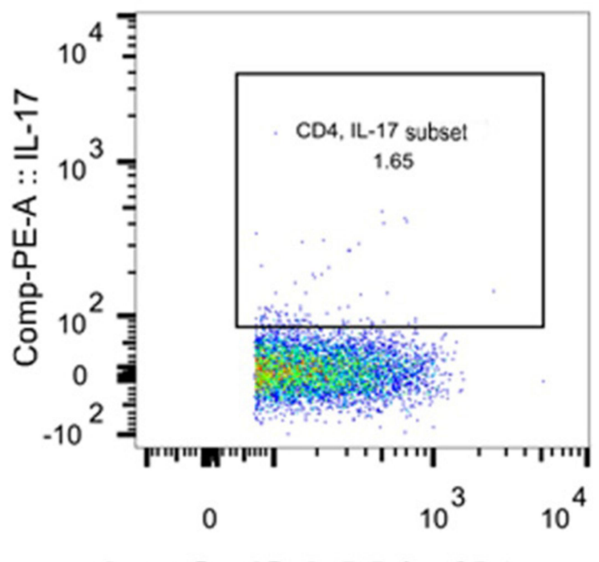

Comp-PerCP-Cy5-5-A ::CD4

Th17
B

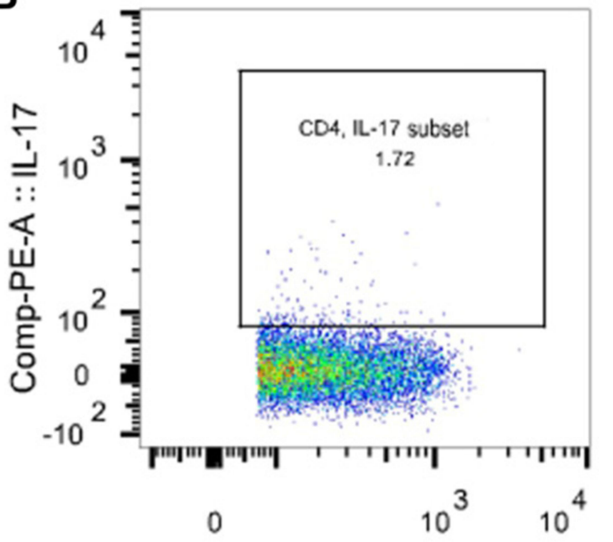

Comp-PerCP-Cy5-5-A ::CD4

D Th17

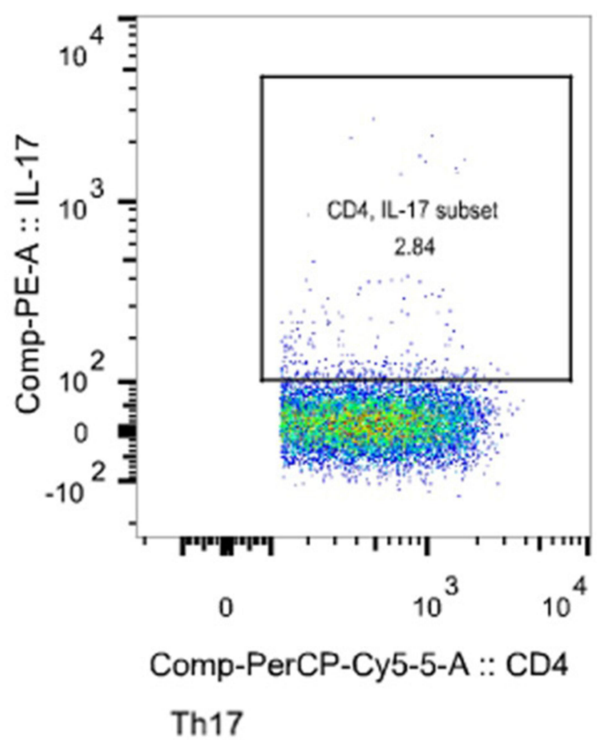

Figure I Representative flow cytometric dot-plots of peripheral blood ThI7 cells in the each group of subjects. (A) is Healthy control; (B) is COPD patient; (C) is T2DM patient; (D) is COPD+T2DM patient. The percentage of positive cells is shown in each panel.

\section{Variation of the Treg/Th I7-Related Inflammatory Cytokines}

Furthermore, the levels of serum IL-17A, IL-17F, IL-21, IL-6, IL-23, TGF- $\beta 1$ and IL-10 in the different groups of subjects were measured for the function of Th17 cells and Tregs. Consistent with changes in Th17 cells, the levels of IL-17A, IL-17F, IL-21, IL-23 and IL-6 in the COPD combined with T2DM patients were significantly higher than that in the healthy controls, COPD and T2DM patients $(\mathrm{P}<0.01)$, levels of IL-17A, IL-17F, IL-21, IL-23 and IL-6 in the COPD and T2DM patients were also significantly higher than that in the healthy controls $(\mathrm{P}<0.01)$. This indicated that the expression of inflammatory cytokines in Th17 cells increased significantly during the pathogenesis of COPD with T2DM. While the levels of TGF- $\beta 1$ and IL-10 in the COPD combined with T2DM patients were significantly lower than those in the healthy controls, COPD and T2DM patients $(\mathrm{P}<0.01)$, levels of TGF- $\beta 1$ and IL-10 in the COPD and T2DM patients were also significantly lower than that in the healthy controls $(\mathrm{P}<0.01)$ (Table 2, Figure 5). This indicates that the expression of treg-related anti-inflammatory cytokines is down-regulated in the pathogenesis of COPD with T2DM.

\section{Discussion}

In this study, we identified the frequency of Th17 and Tregs, the relative levels of ROR- $\gamma \mathrm{t}$ and Foxp 3 mRNA 
A

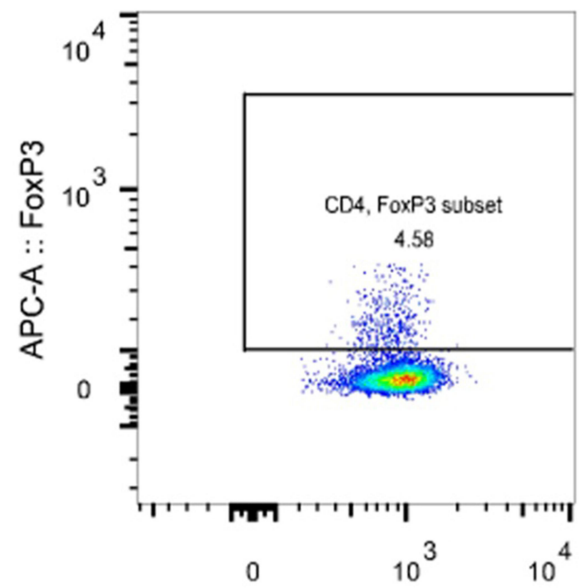

PerCP-Cy5-5-A :: CD4

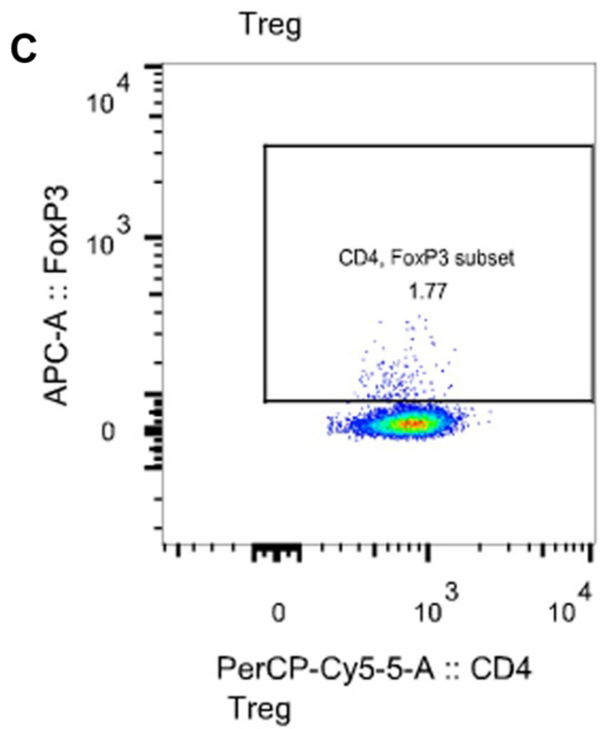

B

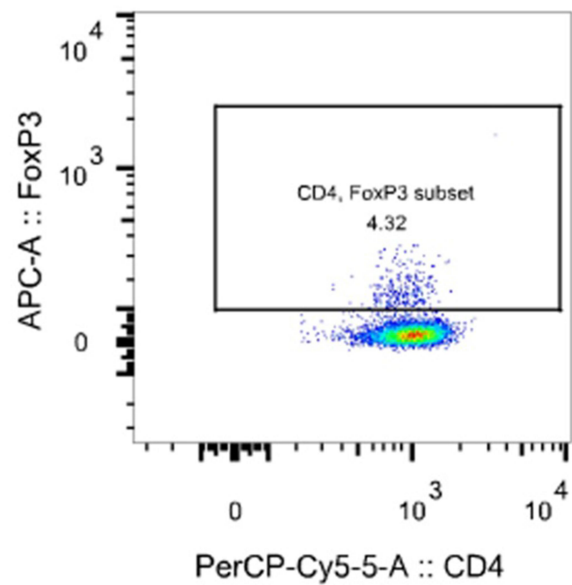

Treg

D

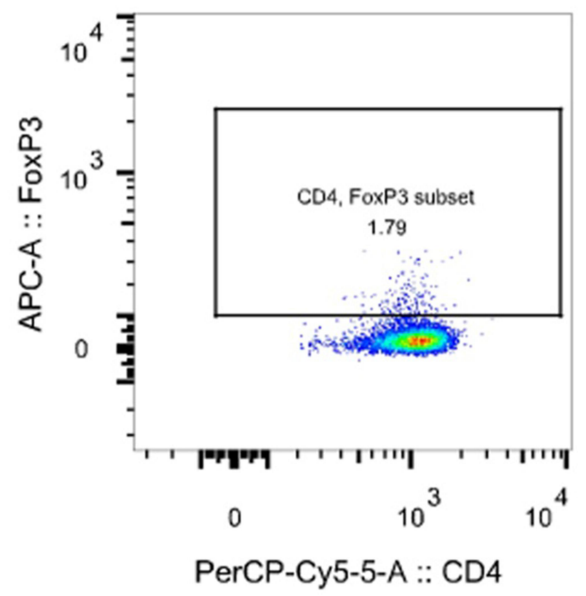

Treg

Figure 2 Representative flow cytometric dot-plots of peripheral blood Tregs cells in the each group of subjects. (A) is Healthy control; (B) is COPD patient; (C) is T2DM patient; (D) is COPD+T2DM patient. The percentage of positive cells is shown in each panel.

transcripts, and the levels of associated serum inflammatory cytokines in the healthy controls, COPD, T2DM and COPD combined with T2DM patients. Our analyses showed that Th17/Treg functional imbalance responses in patients with COPD combined T2DM. It suggests that cellular immunity may play an important role in the pathogenesis of COPD combined with T2DM patients. CD4+ $\mathrm{T}$ cells are crucial for directing immune responses during host defense and for the pathogenesis of inflammatory diseases. CD4 $+\mathrm{T}$ cell subsets, differentiation of Th1, Th2, Th17, Treg and Th9 have been recognized. Th1 cells contribute to the pathogenicity of organ-specific autoimmune diseases such as autoimmune type 1 diabetes, in addition to Th2 cells, IL-9-producing T-helper cells, namely Th9 cells, make up another key T-helper cell subset, which is involved in the pathology of airway inflammation. Previous studies have found that increased frequency of pro-inflammatory Th17 cells in peripheral blood in COPD patients. ${ }^{11,12}$ Tregs can inhibit autoimmune responses by secreting anti-inflammatory cytokines, and were found lower in the COPD patients. ${ }^{13}$ It has been reported that primitive $\mathrm{CD} 4{ }^{+} \mathrm{T}$ tends to differentiate into pro-inflammatory Th17 cells in T2DM. Dysregulation of proinflammatory cells has been observed in rodent models of T2DM and in patients with T2DM, including Th1 and Th17 cells increased, while Treg cells decreased. ${ }^{14,15}$ It is consistent with the results of this study. However, at present, the underlying causes and molecular mechanisms of 

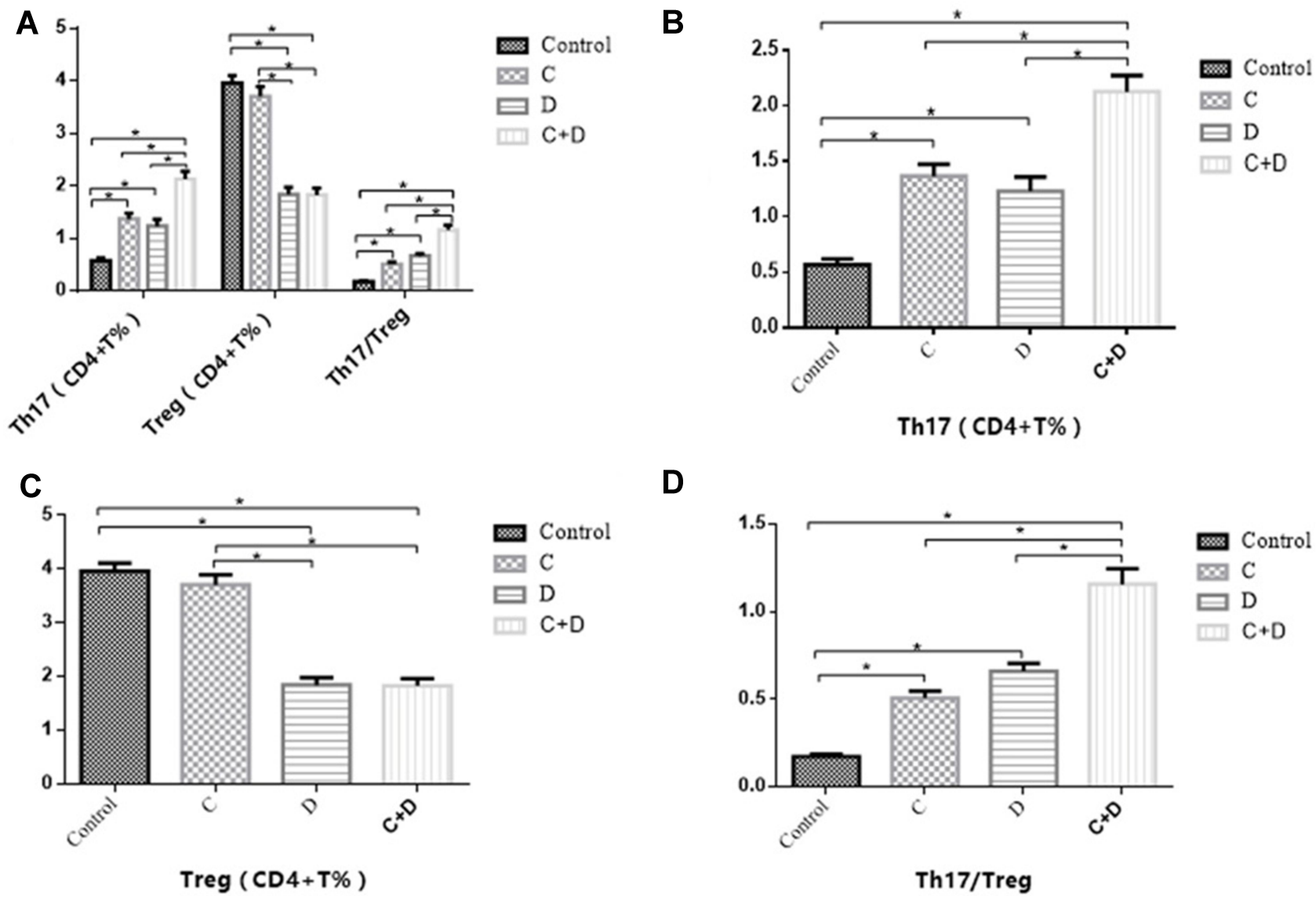

\section{Th17 (CD4+T\%)}

D

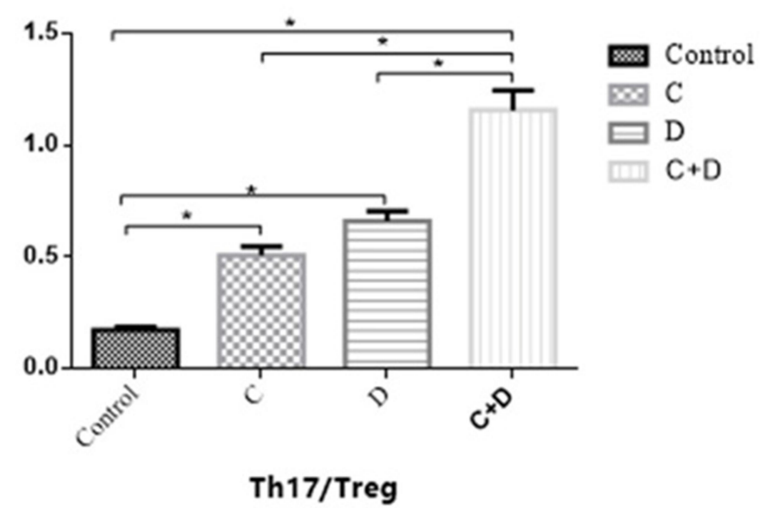

Figure 3 Imbalance of circulating ThI7 and Tregs in COPD, T2DM and COPD combined with T2DM patients. (A) Comparisons of ThI7 and Treg cell percentages in peripheral blood from healthy controls, COPD, T2DM and COPD combined with T2DM patients. (B) Comparisons of ThI7 cell percentages in the 4 groups. (C) Comparisons of Treg cell percentages in the 4 groups. (D) Comparisons of the ratios of ThI7/Treg in the 4 groups. The data are represented as the mean \pm SD; a value of *P $<0.05$ was considered statistically significant.

the changes of Th17 cells and Treg cells in peripheral blood of patients with COPD combined with T2DM are still unclear, relevant studies are relatively lacking, and the basis of Th17/Treg imbalance in patients with COPD combined with T2DM is even more undefined. Studies have shown that the imbalance of Th17 and Treg cells in COPD may be closely related to the persistence of chronic inflammation. Caveolin-1 protein is a key regulatory protein in the pathogenesis of chronic respiratory inflammatory diseases, and Caveolin-1 deficiency can lead to imbalance of Th17 and Treg cell ratio in COPD state. ${ }^{16}$ In the T2DM state, the ratio of anti-apoptotic gene Bcl-2/ Bax to pro-apoptotic gene Bax in Treg cells of patients is lower than that of healthy control, and the imbalance of Bcl-2/Bax may lead to higher sensitivity of Treg cell death than that of healthy control. ${ }^{17}$ Therefore, Increased frequencies of Th17 cells and decreased frequencies of Treg cells are likely mediated by potent pro-inflammatory responses during the pathogenesis of COPD combined with T2DM.

Meanwhile, the same tendency of the relative levels of ROR $\gamma t$ and Foxp3 mRNA transcripts and the levels of IL17A, IL-17F, IL-21, IL-23, IL-10 and TGF $\beta 1$ were also observed in these groups of subjects. These findings indicate that the immune effect mediated by novel cell subsets Th17 and Treg cells is abnormal in patients with COPD complicated with T2DM, and this abnormal immune function may have special potential significance for disease progression. Th17 cells and Treg cells have a related differentiation process, and influence each other's proliferation and activation under certain conditions. Studies have found that Treg cells may undergo gene recombination and reverse into Th17 cells under certain inflammatory environmental conditions. ${ }^{18}$ Low concentration of TGF- $\beta 1$ can promote the expression of ROR $\gamma \mathrm{t}$, while high concentration of TGF- $\beta 1$ can inhibit the function and expression of 
A

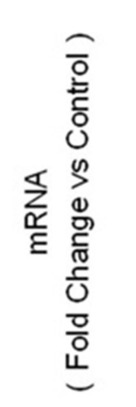

C

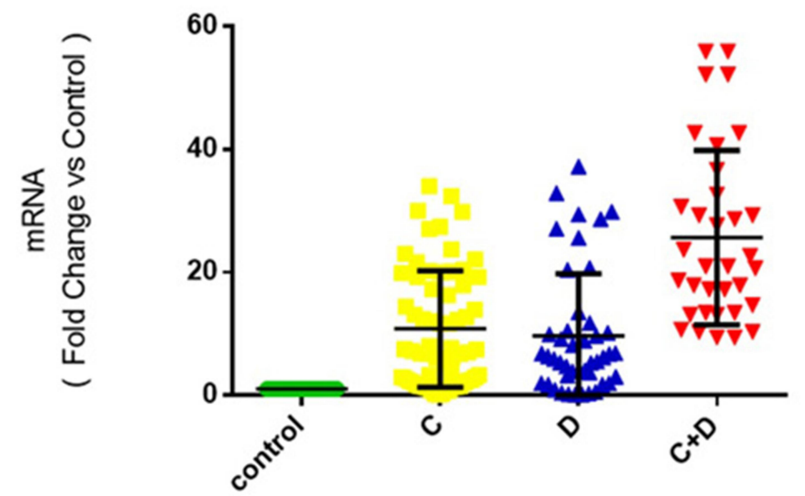

B

Foxp3
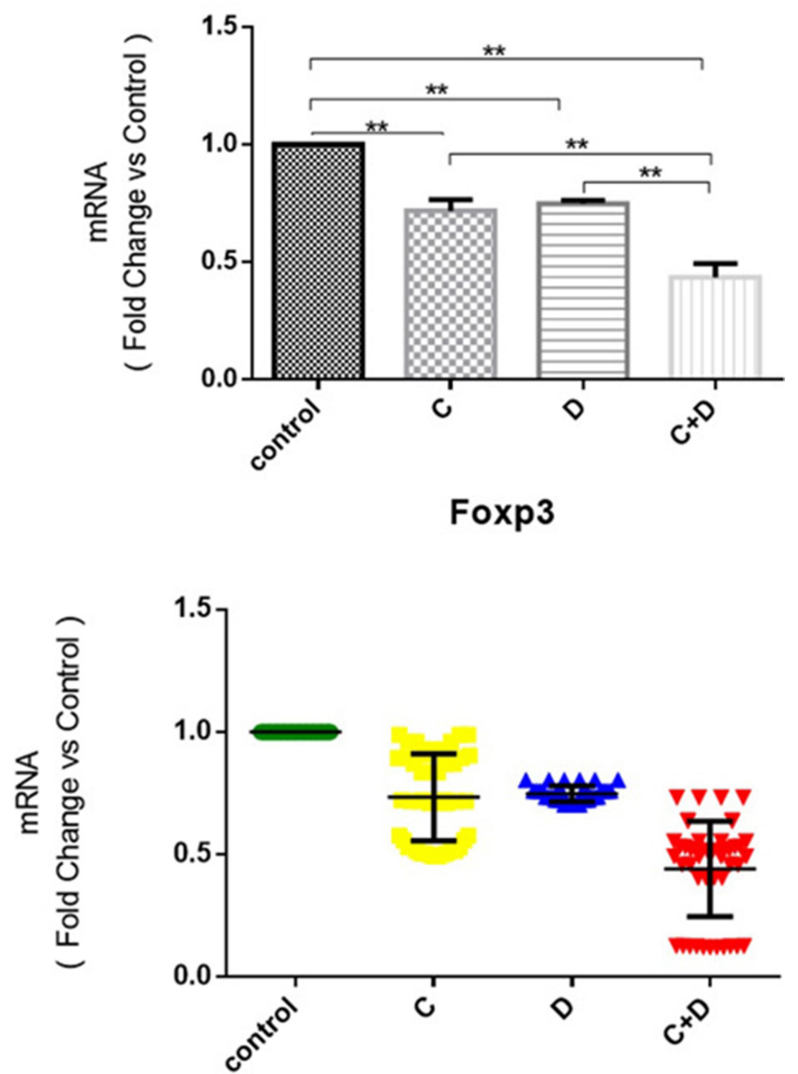

Figure 4 The relative levels of Foxp3 and ROR $\gamma \mathrm{t}$ mRNA transcripts to the control glyceraldehyde 3-phosphate dehydrogenase (GAPDH) in PBMCs from healthy controls, COPD, T2DM and COPD combined with T2DM patients. (A and C) Comparisons of ROR $\gamma \mathrm{t}$ in the 4 groups. (B and $\mathbf{D})$ Comparisons of Foxp 3 in the 4 groups. The data are represented as the mean \pm SD; a value of $* * P<0.0$ I was considered statistically significant. COPD (C group), T2DM (D group) and COPD combined with T2DM (C+D group).

ROR $\gamma$ t, and T cells and even Treg cells themselves can be the source of TGF- $\beta 1 .{ }^{19}$ In this study, we found that patients with COPD combined with T2DM had lower serum TGF- $\beta 1$ levels compared with normal control group and COPD group, which exactly matched the cytokine environment required by the above initial high expression of ROR $\gamma$ t. In addition, IL-21 and TGF- $\beta 1$ coact to up-regulate the expression of ROR $\gamma t$ by phosphorylation of STAT3, and IL-21 can promote the expression of IL-17 in Th17 cells. IL-21 enhances Th17 cell response in an autocrine manner, and overexpresses and maintains the inflammatory response mediated by Th17 cells. Through the IL-21-Th17-IL-21 feedback loop, the differentiation of Th17 cells is continuously induced to stimulate Th17 cells to secrete more IL-21. IL-21 can also upregulate the expression of IL-23R and enhance the response of Th17 cells to IL-23, so as to maintain the stability and proliferation of Th17 cells. ${ }^{20}$ IL- 6 can also down-regulate the activity of Treg cells and further expand the inflammatory response. $\mathrm{CD} 4^{+} \mathrm{T}$ cells differentiated into Treg cells when only TGF- $\beta 1$ was present. And when the TGF- $\beta 1$ and CD4+T cells differentiate into Th17 cells when IL-6 co-exists, and the transformation is mutually exclusive, in which IL-6 plays a key role. ${ }^{21}$ HIF- $1 \alpha$ can form a complex with ROR $\gamma$ t or directly activate ROR $\gamma t$ to promote Th17 cells to secrete IL-17, while HIF- $1 \alpha$ binding to Foxp3 can inhibit the development of Treg cells. Chronic hypoxia in patients with COPD combined with T2DM may cause HIF-1 $\alpha$ to increase and activate ROR $\gamma$ t. $^{22}$ Foxp3 affects homeostasis regulation of the body by regulating transcription and protein expression of Treg cells, and plays an important role in autoimmune diseases, infection and tumor immunity. Natural $\mathrm{CD} 4^{+} \mathrm{CD} 25^{-} \mathrm{T}$ cells transfected by Foxp3 can play an immunosuppressive role, block the proliferation of effector cells, and inhibit the occurrence of inflammatory bowel 
A

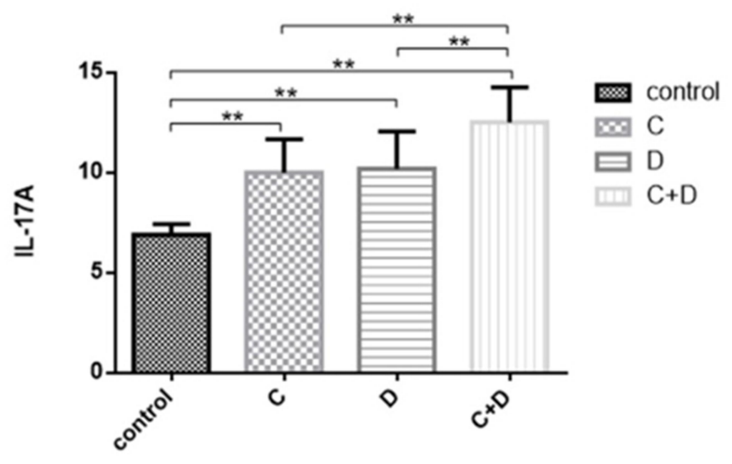

C

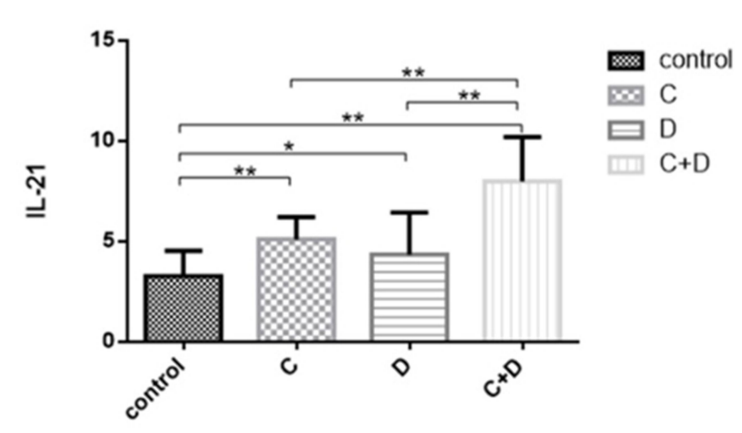

E

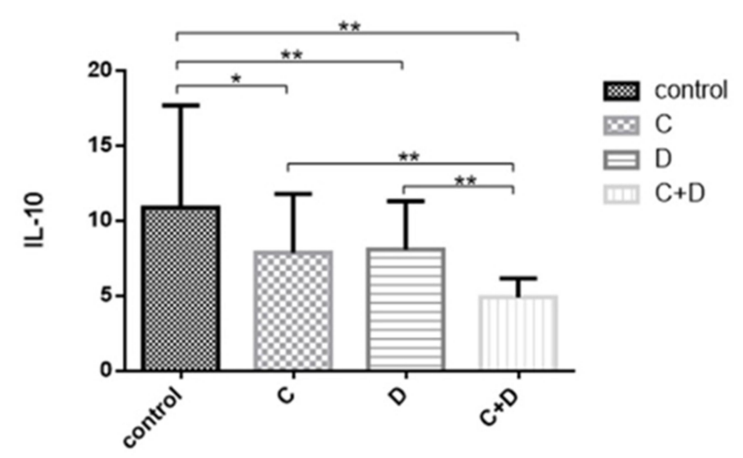

B

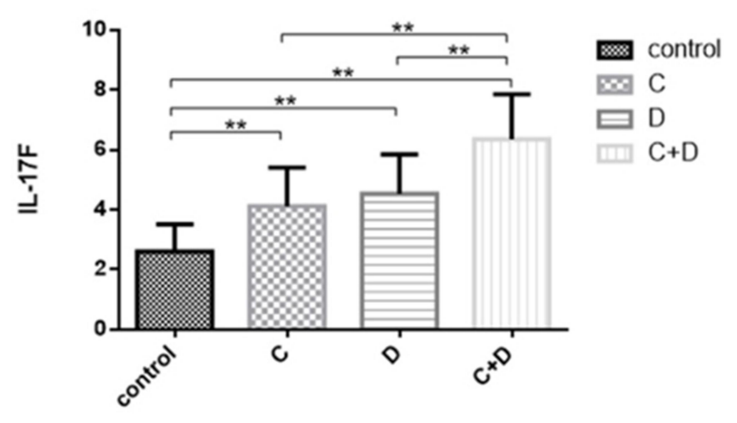

D

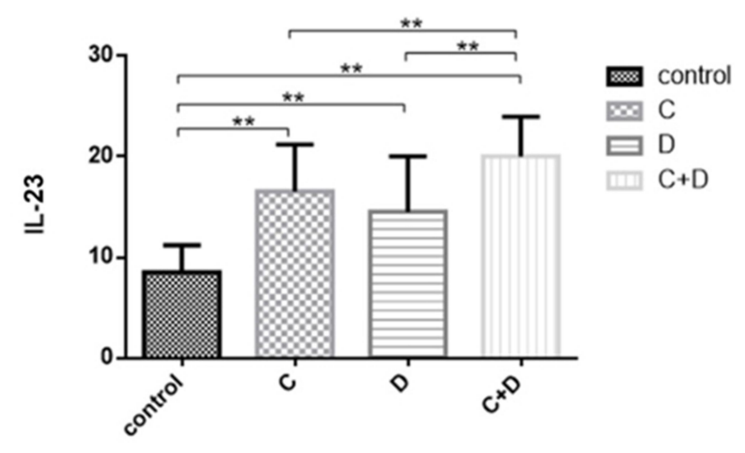

$\mathbf{F}$

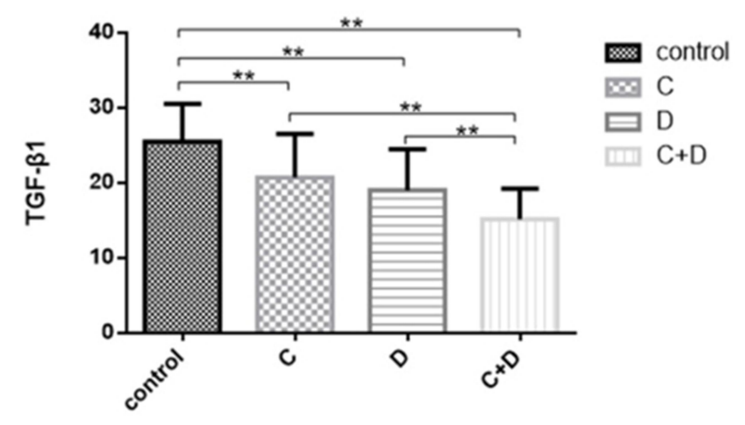

G

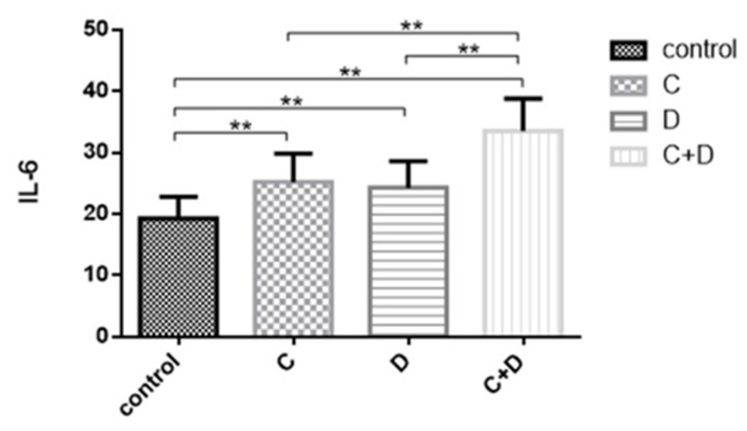

Figure 5 Disordered homeostasis of serum IL-I7A, IL-I7F, IL-2I, IL-23, IL-I0, TGF- $\beta$ I and IL-6 in COPD, T2DM and COPD combined with T2DM patients. Levels of inflammatory cytokines were quantified by ELISA in the 4 groups (A) IL-I7A, (B) IL-I7F, (C) IL-2I, (D) IL-23, (E) IL-I0, (F) TGF- $\beta$ I, (G) IL-6. The data are represented as the mean $\pm \mathrm{SD}$; a value of $* \mathrm{P}<0.05$ or $* * \mathrm{P}<0.01$ was considered statistically significant. COPD (C group), T2DM (D group) and COPD combined with T2DM (C+D group). 
disease and autoimmune gastritis. The deficiency of Foxp3 can cause a variety of autoimmune diseases, such as allergic diseases, diabetes, eczema, lymphocyte proliferation, etc. However, abnormal enhancement of Foxp3 function also leads to tumorigenesis and pathological immunosuppression. ${ }^{23}$ Our results indicate that there are Th17/Treg imbalance in COPD combined with T2DM, suggesting that cellular immunity may play an important role in the pathological process of COPD combined with T2DM, and that Th17/Treg imbalance may be associated with the disease progression and clinical outcome of COPD combined with T2DM.

\section{Conclusion}

Overall, COPD combined with T2DM patients not only has abnormal Th17/Treg cell number, but also has disorder of cellular immune function. The transformation of the balance between Th17/Treg cells to pro-inflammatory Th17 cells can further lead to the accumulation of inflammatory mediators, resulting in a pro-inflammatory positive feedback in the patient, cascading and amplifying the cascading inflammatory response, ultimately lead to uncontrolled and persistent inflammation. Imbalance of Th17/Treg and related cytokines may cause or aggravate tissue damage in patients with COPD combined with T2DM. Therefore, monitoring of cellular immune function in patients with COPD combined with T2DM is of great value for the analysis of clinical conditions, observation of efficacy and judgment of prognosis. We also recognized that our study has limitations, including limited sample size, lack of inflammatory responses tissue samples in the target lung organ and further molecular mechanism investigations. In summary, our findings show that the level of Th17 cell-related pro-inflammatory cytokines increased and the level of Treg cell-related anti-inflammatory cytokines decreased in patients with COPD combined with T2DM, suggesting that there is a Th17/Treg dysfunction in the peripheral blood of patients, and the balance shifts to the proinflammatory direction. Th17/Treg dysfunction may be one of the reasons for the occurrence and development of COPD combined with T2DM.

\section{Acknowledgments}

This study was supported by grants from the Yunnan Province Science and Technology Department-Kunming Medical University Applied Basic Research Joint Special Project-Young Doctoral Program (grant no. 202001AY070001-143) and The First Affiliated Hospital of Kunming Medical University Doctoral Research Fund
Project (grant no. 2018BS016). The authors are grateful to the staff at Second Department of Respiratory Medicine, The First Affiliated Hospital of Kunming Medical University for their help in recruiting patients to the study.

\section{Disclosure}

The authors report no conflicts of interests in this work.

\section{References}

1. Muneswarao J, Verma AK, Hassali Ahmad MA. Global initiative for chronic obstructive lung disease (GOLD) 2018 report: highlighting an incorrect information. Pulm Pharmacol Ther. 2017;49:10. doi:10.1016/j.pupt.2017.12.009

2. Caughey GE, Roughead EE, Vitry AI, et al. Comorbidity in the elderly with diabetes: identification of areas of potential treatment conflicts. Diabetes Res Clin Pract. 2010;87(3):385-393. doi:10.1016/ j.diabres.2009.10.019

3. Cazzola M, Bettoncelli G, Sessa E, et al. Prevalence of comorbidities in patients with chronic obstructive pulmonary disease. Respiration. 2010;80(2):112-119. doi:10.1159/000281880

4. Miller J, Edwards LD, Agusti A, et al. Comorbidity, systemic inflammation and outcomes in the ECLIPSE cohort. Respir Med. 2013;107 (9):1376-1384. doi:10.1016/j.rmed.2013.05.001

5. Crisafulli E 1, Torres A, Huerta A, et al. C-reactive protein at discharge, diabetes mellitus and $\geq 1$ hospitalization during previous year predict early readmission in patients with acute exacerbation of chronic obstructive pulmonary disease. COPD. 2015;12 (3):306-314. doi:10.3109/15412555.2014.933954

6. Cazzola M, Calzetta L, Rogliani P, et al. High glucose enhances responsiveness of human airways smooth muscle via the Rho/ ROCK pathway. Am J Respir Cell Mol Biol. 2012;47(4):509-516. doi:10.1165/rcmb.2011-0449OC

7. Brennan AL, Gyi KM, Wood DM, et al. Airway glucose concentrations and effect on growth of respiratory pathogens in cystic fibrosis. J Cyst Fibros. 2007;6(2):101-109. doi:10.1016/j.jcf.2006.03.009

8. Gläser S, Krüger S, Merkel M, et al. Chronic obstructive pulmonary disease and diabetes mellitus: a systematic review of the literature. Respiration. 2015;89(3):253-264. doi:10.1159/000369863

9. Zhang JC, Chen G, Chen L, et al. TGF- $\beta$ /BAMBI pathway dysfunction contributes to peripheral Th17/Treg imbalance in chronic obstructive pulmonary disease. Sci Rep. 2016;6:31911. doi:10.1038/ srep31911

10. Imani S, Salimian J, Fu J, et al. Th17/Treg-related cytokine imbalance in sulfur mustard exposed and stable chronic obstructive pulmonary (COPD) patients: correlation with disease activity. Immunopharmacol Immunotoxicol. 2016:1-11. doi:10.3109/ 08923973.2015.1127509

11. Vargas-Rojas MI, Ramírez-Venegas A, Limón-Camacho L, Ochoa L, Hernández-Zenteno R, Sansores RH. Increase of Th17 cells in peripheral blood of patients with chronic obstructive pulmonary disease. Respir Med. 2011;105(11):1648-1654. doi:10.1016/j. rmed.2011.05.017

12. Stefano AD, Caramori G, Gnemmi I, et al. T helper type 17-related cytokine expression is increased in the bronchial mucosa of stable chronic obstructive pulmonary disease patients. Clin Exp Immunol. 2010;157(2):316-324. doi:10.1111/j.1365-2249.2009.03965.x

13. Yang X, Huo B, Zhong X, et al. Imbalance between subpopulations of regulatory $\mathrm{T}$ cells in patients with acute exacerbation of COPD. COPD. 2017;14(6):618-625. doi:10.1080/15412555.2017.1385055

14. Dawei L, Jinni C, Xiaolin C, et al. Expression of Th17/Treg cells and related cytokines in peripheral blood of diabetes mellitus patients. Prog Mod Biomed. 2016;16(30):5987. 
15. Korn T, Bettelli E, Gao W, et al. IL-21 initiates an alternative pathway to induce proinflammatory $\mathrm{T}(\mathrm{H}) 17$ cells. Nature. 2007;448 (7152):484-487. doi:10.1038/nature05970

16. Sun N, Wei X, Wang J, et al. Caveolin-1 promotes the imbalance of Th17/Treg in patients with chronic obstructive pulmonary disease. Inflammation. 2016;39(6):2008-2015. doi:10.1007/s10753-0160436-x

17. Zeng C, Shi X, Zhang B, et al. The imbalance of Th17/Th1/Tregs in patients with type 2 diabetes: relationship with metabolic factors and complications. J Mol Med. 2012;90(2):175-186. doi:10.1007/s00109011-0816-5

18. Noack M, Miossec P. Th17 and regulatory T cell balance in autoimmune and inflammatory diseases. Autoimmun Rev. 2014;13 (6):668-677. doi:10.1016/j.autrev.2013.12.004

19. Kumar P, Subramaniyam G. Molecular underpinnings of Th17 immune-regulation and their implications in autoimmune diabetes. Cytokine. 2015;71(2):366-376. doi:10.1016/j.cyto.2014.10.010
20. Lei L, Zhong X-N, He Z-Y, et al. IL-21 induction of CD4+ T cell differentiation into Th17 cells contributes to bleomycin-induced fibrosis in mice. Cell Biol Int. 2015;39(4):388-399. doi:10.1002/ cbin. 10410

21. Jia X, Minghui H, Wang C, et al. Coordinated gene expression of Th17- and Treg-associated molecules correlated with resolution of the monophasic experimental autoimmune uveitis. Mol Vis. 2011;17:1493-1507.

22. Yang HY. Control of T(H)17/T(reg) balance by hypoxia-inducible factor 1. Cell. 2011;146(5):772-784. doi:10.1016/j.cell.2011.07.033

23. Bilate AM, Lafaille J. Induced CD4+Foxp3+ regulatory $\mathrm{T}$ cells in immune tolerance. Annu Rev Immunol. 2012;30(30):733-758. doi:10.1146/annurev-immunol-020711-075043

\section{Publish your work in this journal}

The International Journal of COPD is an international, peer-reviewed journal of therapeutics and pharmacology focusing on concise rapid reporting of clinical studies and reviews in COPD. Special focus is given to the pathophysiological processes underlying the disease, intervention programs, patient focused education, and self management protocols. This journal is indexed on PubMed Central, MedLine and CAS. The manuscript management system is completely online and includes a very quick and fair peer-review system, which is all easy to use. Visit http://www.dovepress.com/testimonials.php to read real quotes from published authors. 